\title{
Salaire minimum, allocations chômage et efficacité du marché du travail
}

\author{
Septembre 2004 - Version finale décembre 2006
}

Frédéric Gavrel et Isabelle Le'bon ${ }^{*}{ }^{* *}$

\section{Introduction}

Notre article s'intéresse aux effets du salaire minimum sur l'efficacité du marché du travail. Le rôle de la législation sur les salaires est envisagé ici sous l'angle de son impact sur l'adéquation entre salariés et emplois qui détermine à son tour la productivité moyenne du travail dans l'économie.

Ce travail s'inscrit donc dans la lignée d'une littérature qui s'est développée depuis la seconde moitié des années quatre-vingt-dix et qui procède au réexamen des implications du salaire minimum. Pendant longtemps, l'inefficience de cette contrainte sur la formation des salaires a été considérée comme une évidence. Cependant, plusieurs travaux empiriques récents ont contribué à remettre en cause cette vision. Parmi ces travaux, les articles de Card et Krueger (Card et Krueger (1994) et (1995)) ont joué un rôle crucial. Ces auteurs ont en effet montré que les augmentations du salaire minimum qui sont intervenues en 1991 (au Texas) et en 1992 (dans le New Jersey), n'ont pas conduit à une baisse mais à une hausse de l'emploi dans un secteur où les employés reçoivent ce minimum, le secteur de la restauration rapide.

Formellement, les nouvelles analyses théoriques sont très proches de l'article précurseur de Stigler (1946) qui montrait que l'impact négatif usuellement attendu de la législation des salaires n'existe plus lorsque les employeurs ont un quelconque pouvoir de marché. Dans certains modèles récents d'équilibre de sous-emploi, les entreprises font également face à une relation croissante entre le salaire et l'emploi. Cependant, cette relation ne provient pas du comportement d'offre de travail (comme dans Stigler) mais

* CERENE, Université du Havre, Faculté des Affaires Internationales, 25 rue Philippe Lebon, BP 420, 76057 Le Havre Cedex, France, frederic.gavrel@univ-lehavre.fr, isabelle.lebon@univ-lehavre.fr.

** Les auteurs remercient Antoine d'Autume. Pieter Gautier, André Zylberberg et le rapporteur anonyme pour leurs remarques et suggestions, mais restent seuls responsables des éventuelles erreurs et insuffisances. 
d'une contrainte d'incitation (Rebitzer et Taylor (1995), Card et Krueger (1995)).

Dans notre article, les implications du salaire minimum sont envisagées dans une perspective assez différente. En utilisant un modèle d'appariement dans lequel les qualifications des travailleurs sont différenciées horizontalement, nous nous concentrons sur la question de la productivité du travail. Nous montrons ainsi que l'introduction puis l'augmentation du salaire minimum améliorent la qualité de l'affectation des emplois aux travailleurs. Pour être viables, les associations entre emplois et travailleurs doivent en effet avoir un rendement supérieur à ce salaire minimum. En rendant les "mauvaises" associations impossibles, le salaire minimum augmente donc nécessairement la productivité moyenne du travail. En d'autres termes, le salaire minimum rend les agents plus exigeants, conduisant ainsi au rejet d'une plus grande partie des appariements. Des simulations numériques permettent de montrer que l'effet de productivité du salaire minimum peut faire augmenter le surplus collectif de l'économie.

La théorie microéconomique récente du chômage a déjà fait apparaître un lien positif entre la productivité (du travail) et les allocations chômage ${ }^{1}$. En effet, une hausse des allocations chômage améliore les opportunités extérieures des chômeurs, les rendant plus sélectifs (Marimon et Zilibotti (1999), Amine, Gavrel et Lebon (2007)). En outre, l'assurance chômage rend les travailleurs susceptibles d'accepter plus facilement un emploi comportant un fort risque de destruction. En supposant que les emplois les plus risqués sont aussi les plus productifs, Acemoglu et Shimer (2000) obtiennent que les allocations améliorent l'affectation des emplois aux travailleurs. Malgré la diminution du nombre des emplois, l'augmentation des allocations peut ainsi accroître l'efficacité du marché du travail.

Cependant, l'existence d'un salaire minimum doit être pris en compte dans le réexamen de l'effet des allocations chômage. Nous envisageons ce réexamen selon deux axes de raisonnement.

Premièrement, nous établissons qu'aussi longtemps que le salaire minimum est assez élevé pour contraindre la formation des salaires (lorsque certains travailleurs touchent effectivement ce minimum légal), l'assurance chômage perd son effet positif sur la productivité. Une augmentation des allocations chômage est alors toujours inefficace, si l'on exclut les situations de sur-emploi. Avec un salaire minimum contraignant, on retrouve les effets conventionnels négatifs de l'assurance chômage.

Ensuite, nous considérons un état du marché du travail dans lequel l'ensemble des salaires se négocient librement. La législation salariale n'est donc pas contraignante et tous les salaires (y compris le plus faible) sont endogènes. Nous utilisons ce point de départ pour comparer les effets respectifs de

Pissarides (1984) est un article fondateur sur cette question. 
deux mesures de politique économique sur l'efficacité du fonctionnement du marché du travail : d'un côté, un accroissement des allocations chômage sans modification du salaire minimum, de l'autre côté, un accroissement du salaire minimum rendant celui-ci contraignant sans changement des allocations chômage. Cette comparaison est réalisée à l'aide de simulations numériques. Dans les deux cas, en dépit de la baisse de l'emploi, l'accroissement de la productivité permet l'augmentation du surplus social. Cependant, la hausse de ce surplus est plus importante avec le salaire minimum, car la perte d'emplois qui en résulte, est plus faible.

Le modèle analytique, un modèle d'appariement avec des fondements microéconomiques, est présenté dans la deuxième section. Les principaux résultats de statique comparative sont ensuite établis dans la section 3 et les simulations numériques proposées dans la section 4.

\section{Cadre analytique}

L'économie se compose de deux ensembles d'agents très nombreux et neutres face au risque : les travailleurs et les entreprises. Les travailleurs sont hétérogènes, les emplois offerts par les entreprises le sont également.

Les travailleurs ont une durée de vie infinie. Les entreprises qui produisent toutes le même bien, peuvent en revanche disparaître à la suite d'un choc économique. Leur risque de destruction, dont la probabilité est notée $s$, est le même à toutes les périodes. Ainsi, les entreprises sont-elles éternellement jeunes comme les ménages de Blanchard et Fisher (1989). En vertu de la loi des grands nombres, une proportion $s$ des entreprises disparaissent à chaque période. Cependant, la règle de libre-entrée de nouvelles entreprises sur le marché stabilise leur nombre à l'état stationnaire. Tous les agents ont le même taux d'actualisation, $r$, et on note $R$ la somme $(1+r)$.

\subsection{Différenciation des qualifications et processus d'embauche}

Pour décrire la différenciation des travailleurs et des emplois, nous nous inspirons du modèle de Salop (1979) à l'instar de Marimon et Zilibotti (1999) et Gavrel (2001).

\subsubsection{Le cercle des qualifications}

Nous supposons ainsi que l'ensemble des travailleurs sont uniformément répartis sur un cercle dont la circonférence est égale à deux (voir Graphique 1). Cette distribution est supposée exogène. La position d'un travailleur sur le cercle ne représente pas sa localisation spatiale ou le niveau de sa productivité 
mais le "type" de sa qualification. La distribution des entreprises autour du cercle est également uniforme. La position d'une entreprise représente son "type", autrement dit la qualification qui correspond parfaitement à ses besoins.

Considérons deux points $A$ et $B$ du cercle. Soit $l$ la longueur du plus court des deux arcs de cercle séparant $A$ et $B(0 \leq l \leq 1)$. Cette distance mesure la différence entre le "type" du travailleur situé au point $A$ (respectivement de l'entreprise) et le "type" de l'entreprise située au point $B$ (respectivement $\mathrm{du}$ travailleur). Ainsi, l'adéquation entre le travailleur et l'entreprise est parfaite lorsque la distance l est égale à zéro, alors que l'inadéquation est maximale lorsgue $l$ atteint un.

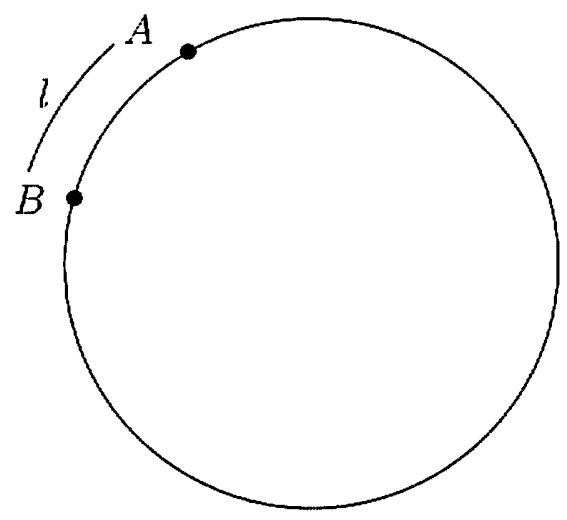

Figure 1 : Le cercle des qualifications

Dans ce modèle, la productivité d'un travailleur est décroissante en fonction de la distance $l$ qui sépare sa qualification des besoins de l'entreprise qui l'emploie. Soit $y(l)$ cette productivité. Selon une hypothèse standard en économie industrielle, on retient une "fonction d'adéquation" $y(l)$ concave $\left(y^{\prime}(l)<0, y^{\prime \prime}(l) \leq 0\right)$.

Chaque entreprise emploie un travailleur unique. La productivité de ce travailleur détermine donc la production de l'entreprise.

\subsubsection{Le processus d'embauche}

Pour pouvoir être considérée comme viable, l'association entre un emploi et un travailleur ne peut pas être "trop mauvaise". En d'autres termes, la distance $l$ entre leurs types respectifs doit être inférieure à la désadéquation limite que nous notons $\lambda$. A ce stade de l'analyse, la désadaquation limite est considérée comme donnée, nous l'endogénéiserons ultérieurement en étudiant le processus de formation des salaires. 
Contrairement à Marimon et Zilibotti (1999) dont la fonction d'embauche est ad hoc, nous déduisons la notre d'une extension du modèle "urnes-boules" ("urn-ball model") (Hall (1977), Pissarides (1979), Petrongolo et Pissarides (2001)). Notons que, comme Marimon et Zilibotti (1999), nous excluons le on-the-job-search, i.e. que les travailleurs déjà dotés d'un emploi puissent continuer à en chercher un meilleur.

Conformément à la théorie de la recherche d'emploi (voir par exemple Mc Kenna (1985)) et dans une optique de simplification, nous supposons que les chômeurs n'émettent qu'une seule candidature par période ${ }^{2}$. Cependant, les chômeurs ont une certaine information a priori sur les emplois offerts. Plus précisément, nous supposons que l'emploi vacant auquel un chômeur candidate est choisi au hasard parmi ceux qui sont situés à une distance inférieure à $x$ par rapport à son propre type. La variable $x(0 \leq x \leq 1)$ est une exogène qui représente la transparence $d u$ marché du travail ou l'information sur le marché du travail. Plus la valeur de $x$ est faible et plus le marché du travail est transparent. Lorsque $x$ est. égal à zéro, la recherche est parfaitement orientée, et il n'y a aucune désadéquation entre les besoins des entreprises et les caractéristiques de leur salarié.

Soit $U$ le nombre de chômeurs et $V$ le nombre d'emplois vacants. La tension sur le marché du travail s'écrit alors $\theta=\frac{V}{V}$. On peut ainsi montrer (voir annexe A) que la probabilité de pourvoir un emploi vacant, notée $q$, est déterminée par la relation suivante :

$$
q=1-e^{-\frac{\lambda}{x \theta}}
$$

En effet, pour pourvoir un emploi vacant, une entreprise n'a besoin de rencontrer qu'un seul travailleur employable, i.e. un travailleur dont le type n'est pas situé à une distance supérieure à $\lambda$ de celui de l'entreprise. Concernant le comportement de recrutement des entreprises, la fonction d'appariement retenue revient à supposer qu'elles procèdent à un tirage aléatoire (sans remise) dans l'ensemble de leurs candidats jusqu'à trouver un travailleur employable (si tant est qu'un tel candidat existe) ; ce travailleur est alors embauché ${ }^{3}$. Cette hypothèse est conforme à celle faite implicitement par Marimon et Zilibotti (1999).

L'équation (1) montre qu'une plus grande exigence de la part des entreprises, (i.e. une baisse de $\lambda$ ) réduit la probabilité de pourvoir un emploi vacant de la même façon qu'une hausse du nombre d'emplois vacants ou une baisse du chômage (i.e. une hausse de la tension sur le marché du travail $\theta$ ).

Le nombre total des embauchés, noté $H$, se déduit de $q$ :

2 Voir Albrecht, Gautier et Vroman (2003) pour une extension du "urn-ball moder" dans laquelle les travailleurs qui sont homogènes, contactent plusieurs employeurs à chaque période.

3 Gavrel et Lebon (2004) traitent le cas où les entreprises examinent toules les candidatures afin de choisir te "meilleur" des candidats acceptables. 


$$
H=\left(1-e^{-\frac{\lambda}{x \theta}}\right) V
$$

La fonction d'embauche, ainsi obtenue, est à rendements d'échelle constants, ce qui est compatible avec les résultats des études empiriques (Blanchard et Diamond (1989)).

La probabilité d'embauche des travailleurs, $p$, est obtenue en divisant le nombre des travailleurs embauchés, $H$, par le niveau du chômage, $U$ :

$$
p=\theta\left(1-e^{-\frac{\lambda}{x \theta}}\right)
$$

La probabilité $p$ est une fonction croissante de la désadéquation limite, $\lambda$, et une fonction décroissante de la distance, $x$. Dans, l'annexe $\mathrm{B}$, nous établissons que $p$ est également en relation croissante avec la tension sur le marché du travail, $\theta$.

Ainsi, une augmentation de la désadéquation limite, $\lambda$, (i.e. une moindre sélectivité) augmente à la fois $p$ et $q$. Cependant, cette augmentation qui diminue la production moyenne par entreprise, $\bar{y}$, peut s'avérer socialement indésirable. Par ailleurs, un accroissement de la distance $x$ (i.e. une moindre transparence du marché du travail) réduit ces deux probabilités de transition.

\subsection{Utilités et profits intertemporels}

\subsubsection{Les travailleurs}

Les travailleurs employés par une entreprise ont une productivité qui dépend de la distance $l$ séparant les caractéristiques de leur emploi de leurs propres caractéristiques ; leur salaire $w(l)$ dépend alors lui aussi de cette distance. Il en est donc de même de leur espérance d'utilité intertemporelle $W_{E}(l)$.

L'espérance d'utilité intertemporelle des chômeurs, $W_{U}$, est principalement déterminée par la désadéquation limite, $\lambda$, la distance en dessous de laquelle un couple employeur/employé engendre un surplus positif pour un salaire qui n'est pas inférieur au minimum légal. $\lambda$ affecte en effet le taux d'embauche des chômeurs, $p$, et l'espérance d'utilité moyenne d'un travailleur qui trouve un emploi, notée $\bar{W}_{E}$.

Etant donnée l'uniformité de la distribution des emplois vacants, l'espérance conditionnelle $\bar{W}_{E}$ vérifie la relation suivante :

$$
\bar{W}_{E}=\frac{1}{\lambda} \int_{0}^{\lambda} W_{E}(l) d l
$$


A l'état stationnaire, les utilités intertemporelles $W_{E}(l)$ et $W_{U}$ s'écrivent respectivement :

$$
\begin{gathered}
W_{E}(l)=w(l)+R^{-1}\left[s W_{U}+(1-s) W_{E}(l)\right] \\
W_{U}=\zeta+R^{-1}\left[p \bar{W}_{E}+(1-p) W_{U}\right]
\end{gathered}
$$

où $\zeta$ représente le revenu d'un chômeur (production domestique plus allocations chômage).

\subsubsection{Les entreprises}

Les emplois offerts par les entreprises sont soit vacants soit occupés. Plus précisément, la valeur d'un emploi occupé, notée $J_{F}(l)$, dépend évidemment de la distance $l$ qui sépare les types de l'employeur et de l'employé. Soit $J_{V}$ la valeur espérée d'un emploi vacant. La valeur d'un emploi occupé, $J_{F}(l)$, est donnée par :

$$
J_{F}(l)=y(l)-w(l)+R^{-1}\left[s J_{V}+(1-s) J_{F}(l)\right]
$$

La valeur d'un emploi vacant est une fonction de la désadéquation limite $\lambda$. En effet, cette limite affecte la probabilité, $q$, de pourvoir l'emploi ainsi que l'espérance de la valeur de l'emploi une fois occupé. Cette dernière espérance conditionnelle, notée $\overline{J_{F}}$, vérifie la relation suivante :

$$
\overline{J_{F}}=\frac{1}{\lambda} \int_{0}^{\lambda} J_{F}(l) d l
$$

La valeur d'un emploi vacant peut ainsi s'écrire sous la forme :

$$
J_{V}=-c+R^{-1}\left[q \bar{J}_{F}+(1-q) J_{V}\right]
$$

où $c$ représente le coût (d'annonce et de capital) d'un emploi vacant.

Comme dans les modèles d'appariement usuels (Pissarides (2000)), nous supposons que les entreprises entrent librement sur le marché. Ainsi de nouveaux emplois vacants sont-ils créés tant que leur valeur reste positive. Il en résulte que :

$$
J_{V}=0
$$

$\bar{y}$ et $\bar{w}$ représentent respectivement la production moyenne et le salaire moyen :

$$
\begin{aligned}
& \bar{y}=\frac{1}{\lambda} \int_{0}^{\lambda} y(l) d l \\
& \bar{w}=\frac{1}{\lambda} \int_{0}^{\lambda} w(l) d l
\end{aligned}
$$

Etant données les équations (7), (8) et (9), la condition de libre-entrée (10) implique :

$$
J_{F}=\frac{R(\bar{y}-\bar{w})}{r+s}
$$


et

$$
\overline{J_{F}}=\frac{R C}{q}
$$

\subsection{La négociation salariale et la désadéquation limite}

Conformément aux modèles d'appariement usuels, nous supposons que le résultat de la négociation salariale est obtenu à partir de la maximisation de l'objectif de Nash généralisé sous contrainte que le salaire obtenu ne soit pas inférieur au salaire minimum de l'économie, noté $m$. Soit $\beta$ le pouvoir de négociation des travailleurs. Le programme d'optimisation s'écrit de la façon suivante :

$$
\begin{gathered}
\operatorname{Max}\left(W_{E}(l)-W_{U}\right)^{\beta}\left(J_{F}(l)-J_{V}\right)^{(1-\beta)} \\
\text { s.c : } w(l) \geq m
\end{gathered}
$$

Ce problème va donc admettre deux types de solution en fonction des niveaux relatifs du revenu des chômeurs $(\zeta)$ et du minimum légal $(m)$.

Lorsque le salaire minimum légal est faible relativement au revenu des chômeurs, la contrainte n'est jamais saturée. Dans ce premier cas, le surplus total est simplement réparti selon la règle de Nash :

$$
W_{E}(l)-W_{U}=\beta\left[W_{E}(l)+J_{F}(l)-W_{U}-J_{V}\right]
$$

à condition que l'association entre l'employeur et l'employé soit viable, ce qui est le cas si (et seulement si) elle crée un surplus total positif :

$$
W_{E}(l)+J_{F}(l)-W_{U}-J_{V} \geq 0
$$

La formation des salaires est alors non contrainte, et la désadéquation limite $\lambda$ est déterminée par l'annulation du surplus total.

A l'opposé, si le minimum légal est élevé relativement au revenu des chômeurs, cette contrainte légale va effectivement peser sur la formation des salaires et le fonctionnement de l'économie, rendant les "mauvaises" associations (i.e. les valeurs importantes de $l$ ) impossibles.

Dans ce second cas, nous devons distinguer deux régimes en fonction de la désadéquation, $l$, entre les types de l'employeur et de l'employé. Soit $\hat{l}$ le niveau de désadéquation pour lequel le salaire qui résulte de la règle de Nash (équation (15)), est égal au salaire minimum légal, $m$ :

- Lorsque la désadéquation $l$ est inférieure à la valeur $\hat{l}(l \leq \hat{l})$, le salaire reste déterminé par la règle de Nash.

- Au contraire, lorsque la désadéquation $l$ est supérieure à la valeur $\hat{l}(l>\hat{l})$, on se trouve dans un second régime. En effet, la règle de Nash engendre alors un salaire inférieur au minimum légal, et c'est ce salaire minimum qui est alors appliqué pour fixer la rémunération du travailleur. 
La distance $\hat{l}$ qui marque le passage entre les deux régimes, sera ainsi désignée par le terme de désadéquation de transition.

Dans le second régime $(l>\hat{l})$, lorsque l'employé reçoit le minimum $m$, le profit courant de l'entreprise est donné par $(y(l)-m)$. Cependant, afin que la valeur de l'emploi occupé $J_{F}(l)$ reste positive, ce profit courant doit aussi être positif. Cette condition va alors déterminer la désadéquation limite, $\lambda$, qui ne dépend plus que de ce salaire minimum:

$$
y(\lambda)=m
$$

Il en résulte que le salaire minimum légal procure au gouvernement un instrument de politique économique très efficace pour contrôler la désadéquation sur le marché du travail et la productivité moyenne des entreprises.

\subsection{L'équilibre des flux sur le marché du travail}

A l'équilibre stationnaire, le flux des travailleurs qui trouvent un emploi est égal au flux des travailleurs qui perdent le leur.

Soit $u$ le taux de chômage, la condition d'équilibre des flux s'écrit :

$$
p u=s(1-u)
$$

A partir de cette condition d'équilibre des flux, nous déduisons le taux de chômage $u$ comme une fonction de la probabilité d'embauche :

$$
u=\frac{s}{s+p}
$$

\subsection{La résolution du modèle}

Nous allons tout d'abord étudier la question de la formation des salaires en fonction de la productivité du couple employeur/employé et donc de son niveau de désadéquation. En particulier, nous nous intéresserons au niveau de productivité pour lequel la libre formation du salaire donne un salaire égal au minimum légal. Ce niveau de productivité correspond en effet à la désadéquation de transition qui marque la séparation entre fixation libre et fixation contrainte du salaire. Nous établirons ainsi une première relation entre cette désadéquation de transition et la tension sur le marché du travail. Nous déduirons ensuite du processus de création des emplois vacants une seconde relation entre les mêmes variables, la combinaison de ces deux relations nous donnant l'équilibre sur le marché du travail.

\subsubsection{Formation des salaires, désadéquation de transition et tension sur le marché du travail}

Nous savons que tous les salaires supérieurs au minimum $m$ satisfont la règle de Nash. Pour $l \leq \hat{l}$, l'équation (15) peut être réécrite de la façon suivante : 


$$
(1-\beta)\left[W_{E}(l)-W_{U}\right]=(1-\beta)\left[\left(W_{E}(l)-\bar{W}_{E}\right)+\left(\bar{W}_{E}-W_{U}\right)\right]=\beta J_{F}(l)(20)
$$

Les différences $\left(W_{E}(l)-\bar{W}_{E}\right)$ et $\left(\bar{W}_{E}-W_{U}\right)$ s'expriment à partir des équations (5) et (6) :

$$
\begin{aligned}
W_{E}(l)-\bar{W}_{E} & =\frac{R(w(l)-\bar{w})}{r+s} \\
\bar{W}_{E}-W_{U} & =\frac{R(\bar{w}-\zeta)}{r+s+p}
\end{aligned}
$$

Etant données ces relations et les équations (7) et (10), l'équation (20) se réécrit de la façon suivante (pour tous les $l \leq \hat{l}$ ) :

$$
(1-\beta)\left[\frac{w(l)-\bar{w}}{r+s}+\frac{\bar{w}-\zeta}{r+s+p}\right]=\beta \frac{y(l)-w(l)}{r+s}
$$

Soit $\overline{\hat{w}}$ le salaire moyen et $\overline{\hat{y}}$ la productivité moyenne déterminés sur l'intervalle $[0, \hat{l}], \quad \overline{\hat{w}}=\frac{1}{\imath} \int_{0}^{\hat{l}} w(l) d l$ et $\overline{\hat{y}}=\frac{1}{\hat{l}} \int_{0}^{\hat{l}} y(l) d l$. Par définition de la variable $\bar{w}$ (équation (12)), on peut écrire :

$$
\bar{w}=\frac{\lambda-\hat{l}}{\lambda} m+\frac{\hat{l}}{\lambda} \overline{\hat{w}}
$$

L'équation (21) étant vérifiée pour la distance $\hat{l}$ et puisque $w(\hat{l})=m$, on $\mathrm{a}$ :

$$
(1-\beta)\left[\frac{m-\bar{w}}{r+s}+\frac{\bar{w}-\zeta}{r+s+p}\right]=\beta \frac{y(\hat{l})-m}{r+s}
$$

Cette dernière équation relie trois variables endogènes qui sont la probabilité d'embauche $p$, le salaire moyen $\bar{w}$ et la désadéquation de transition $\hat{l}$. Ainsi, la désadéquation de transition, $\hat{l}$, apparaît-elle comme une fonction implicite de $p$ et $\bar{w}$, et les dérivées partielles de cette fonction implicite par rapport à $p$ et $\bar{w}$ sont toutes deux positives. Une augmentation de $p$ (ou une augmentation de $\bar{w}$ ) correspond en effet à un accroissement de tous les salaires négociés. En conséquence, la règle de Nash s'applique alors aux salaires de couples de productivité plus faible, d'où une hausse de la désadéquation de transition $\hat{l}$. Un plus grand nombre de travailleurs reçoivent donc un salaire supérieur au minimum légal $m$.

L'intégration de l'équation(21) sur $[0, \hat{l}]$ donne :

$$
(1-\beta)\left[\frac{\overline{\hat{w}}-\bar{w}}{r+s}+\frac{\bar{w}-\zeta}{r+s+p}\right]=\beta \frac{\overline{\hat{y}}-\overline{\hat{w}}}{r+s}
$$

La différence entre les équations (24) et (23) permet alors d'écrire :

$$
\overline{\hat{w}}=m+\beta(\overline{\hat{y}}-y(\hat{l}))
$$


En tenant compte de l'équation (22), nous obtenons :

$$
\bar{w}=m+\frac{\hat{l}}{\lambda} \beta(\overline{\hat{y}}-y(\hat{l}))
$$

Les deux relations précédentes soulignent le fait que les différences entre les salaires négociés $(l \leq \hat{l})$ ne résultent que des variations de la productivité $y(l)$, les opportunités extérieures $W_{U}$ et $J_{V}$ étant les mêmes pour tous les travailleurs et toutes les entreprises. Le salaire moyen de l'économie se déduit alors des niveaux de productivité et de la valeur du minimum salarial.

De plus, il est facile de voir que la différence $(\overline{\hat{y}}-y(\hat{l}))$ est une fonction croissante de la désadéquation de transition $\hat{l}$. Cela résulte de la concavité de $y(l)$. L'équation (26) fait donc apparaître le salaire moyen $\bar{w}$ comme une fonction croissante de la désadéquation de transition $\hat{l}$.

La substitution de l'expression (26) dans l'équation (23) nous permet finalement d'obtenir une première relation liant les variables endogènes $\hat{l}$ et $\theta$ :

$$
(r+s)[m-(1-\beta) \zeta]=\beta\left[(r+s) y(\hat{l})+p(y(\hat{l})-m)+(1-\beta) p \frac{\hat{l}}{\lambda}(\overline{\hat{y}}-y(\hat{l}))\right]
$$

Puisque la probabilité d'embauche $p$ est une fonction (croissante) de la tension $\theta$, l'équation (27) détermine la désadéquation de transition $\hat{l}$ comme une fonction implicite de la tension sur le marché du travail :

$$
\hat{l}=W S(\theta, .)
$$

La dérivée de $W S($.$) par rapport à \theta$ est positive. Dans l'espace $(\theta, \hat{l})$, cette relation est donc décrite par une courbe croissante WS (Graphique 2).

A productivité donnée, les salaires négociés augmentent avec la tension sur le marché du travail ; cela repousse la désadéquation de transition à des distances plus élevées et donc à des niveaux de productivité plus faibles pour un même salaire minimum.

\subsubsection{Création d'emplois vacants et équilibre}

Afin d'obtenir une deuxième relation entre les variables $\hat{l}$ et $\theta$, nous nous intéressons maintenant au processus de création des emplois vacants. Ce processus est retracé par les équations (13) et (14). Leur combinaison nous donne :

$$
q(\bar{y}-\bar{w})=(r+s) c
$$

En substituant $\bar{w}$ par son expression dans l'équation (26), on obtient :

$$
q\left[\bar{y}-m-\frac{l}{\lambda} \beta(\overline{\hat{y}}-y(\hat{l}))\right]=(r+s) c
$$

Puisque la probabilité $q$ est une fonction (décroissante) de la tension sur le marché du travail $\theta$, l'équation (30) détermine la désadéquation de 
transition $\hat{l}$ comme une fonction implicite de la tension sur le marché du travail :

$$
\hat{l}=V C(\theta, .)
$$

On peut vérifier que la dérivée de la fonction $V C()$. par rapport à $\theta$ est négative. Dans l'espace $(\theta, \hat{l})$, cette relation est donc décrite par une courbe décroissante ( $V C$ ) (Graphique 2).

En effet, le salaire moyen $\bar{w}$ augmentant avec la désadéquation de transition, une hausse de cette désadéquation est donc associée à une diminution de l'ouverture de nouveaux emplois vacants qui se traduit par une baisse de la tension sur le marché du travail.

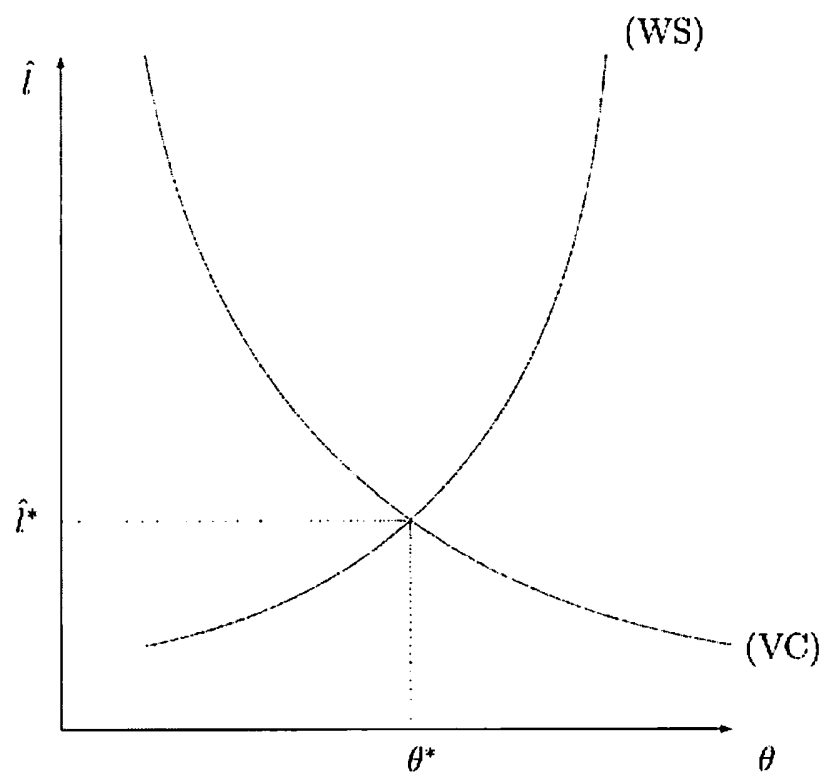

Figure 2: L'équilibre stationnaire

La combinaison des fonctions $W S$ et $V C$ nous permet de déterminer les valeurs d'équilibre de la désadéquation de transition et de la tension sur le marché du travail $\left(\hat{l}^{*}, \theta^{*}\right)$ (voir Graphique 2). A partir de $\hat{l}^{*}$ et $\theta^{*}$, on peut obtenir les valeurs d'équilibre des autres variables du marché du travail.

\section{Statique comparative}

Le tableau 1 retrace les effets des principales exogènes sur les variables du marché du travail. 


\begin{tabular}{|l|c|c|c|c|c|c|c|c|}
\hline & $\lambda$ & $\bar{y}$ & $\theta$ & $\hat{\imath}$ & $\bar{u}$ & $\boldsymbol{p}$ & $\boldsymbol{q}$ & $\boldsymbol{u}$ \\
\hline$x$ & 0 & 0 & $?$ & - & - & - & - & + \\
\hline$s$ & 0 & 0 & - & $?$ & $?$ & - & + & + \\
\hline$\zeta$ & 0 & 0 & - & + & + & - & + & + \\
\hline$m$ & - & + & $?$ & - & $?$ & $?$ & $?$ & $?$ \\
\hline
\end{tabular}

Tableau 1 : Statique comparative

\subsection{Information sur le marché du travail}

Une meilleure information sur le marché du travail, i.e. une plus grande transparence, se traduit par une baisse du paramètre $x$. Lorsque le salaire minimum est effectivement perçu par les salariés les moins productifs de l'économie, une amélioration de la transparence du marché du travail est sans effet sur la désadéquation limite, $\lambda$; elle n'est donc pas susceptible d'engendrer le moindre effet sur la productivité moyenne de l'économie. En revanche, l'amélioration des probabilités de transition, $q$ et $p$, qui résulte directement de cette meilleure information, réduit le chômage et induit une pression à la hausse des salaires négociés. Il en résulte que la proportion des employés recevant le salaire minimum diminue, ce qui se traduit par une hausse de la distance $\hat{l}$. Par contre, l'effet sur la tension sur le marché du travail reste indéterminé.

\subsection{Taux de destruction des emplois}

Le progrès technique peut accélérer le processus de création-destruction des entreprises (voir par exemple Aghion et Howitt (1992)), une hausse du taux de destruction $s$ peut ainsi être interprétée comme résultant d'une telle accélération.

Comme dans l'analyse précédente des effets de la transparence du marché du travail, le taux de destruction ne peut pas affecter la désadéquation limite $\lambda$, et donc la productivité moyenne, qui sont exclusivement liées à la valeur du salaire minimum. La réduction de la durée de survie moyenne d'une entreprise productive entraîne une réduction de la création de nouvelles entreprises, d'où une baisse de la tension sur le marché du travail. Cette diminution de $\theta$ engendre une hausse de $q$ et la baisse de $p$ à $\lambda$ donné. La baisse de $p$ et l'augmentation de $s$ se conjuguent pour provoquer une hausse du taux de chômage. 


\subsection{Les allocations chômage}

Quand tous les salaires sont négociés librement, le changement des coûts de recherche modifie l'affectation des travailleurs aux emplois (Gavrel (2001), Marimon et Zilibotti (1999)). En particulier, une augmentation des allocations chômage (i.e. une baisse des coûts de recherche des chômeurs) améliore cette affectation en rendant les travailleurs plus exigeants dans leur choix. En conséquence, en dépit de la baisse du nombre d'emplois qu'elles provoquent, les allocations chômage peuvent être efficaces.

Dans ce modèle, la désadéquation limite est déterminée par le salaire minimum ; les coûts de recherche perdent alors leur effet de productivité. La hausse de l'ensemble des salaires négociés n'étant compensée par aucune hausse de la productivité, il en résulte une diminution de la profitabilité des entreprises productives qui engendre une diminution des créations d'emplois. Ce comportement conduit à une diminution de la tension sur le marché du travail qui provoque une hausse du taux de chômage.

Les effets positifs d'une hausse des allocations chômage sur le fonctiomement du marché du travail sont donc étroitement liés à l'absence de salaire minimum contraignant la formation des salaires ${ }^{4}$. Ce constat limite nettement la portée pratique de ce résultat, car la législation du travail de la majorité des pays de l'OCDE comporte un salaire minimum qui est effectivement appliqué à une partie des salariés.

\subsection{Le salaire minimum}

Une augmentation du salaire minimum entraîne un accroissement direct de la productivité minimale possible pour les couples employeur/employé. La hausse de $m$ provoque donc une diminution de la désadéquation limite, $\lambda$, et une augmentation de la productivité moyenne des entreprises, $\bar{y}$.

Une augmentation du salaire minimum $m$ diminue nécessairement la désadéquation de transition, $\hat{l}$. En effet, des salariés à productivité plus forte, dont les salaires étaient précédemment librement négociés, sont "rat,trapés" par le salaire minimum.

Les autres effets du salaire minimum sont indéterminés. Afin de pouvoir apprécier l'effet de son introduction sur l'efficacité du marché du travail, nous allons recourir à une analyse quantitative.

4 Cette conclusion suppose cependant que l'économie se trouve dans une situation de sous-emploi. En cas de sur-emploi, les allocations chômage sont à l'inverse susceptibles d'être combinées de façon efficace avec le salaire minimum, jusqu'à permettre à l'économie d'atteindre une situation d'optimum de premier rang. 


\section{Simulations numériques}

Notre analyse quantitative poursuit un double objectif :

1. Il s'agit tout d'abord de préciser les effets du salaire minimum légal sur l'ensemble des variables endogènes de l'économie lorsque ceux-ci ne peuvent etre établis analytiquement. Ces simulations numériques ne constituent donc pas une calibration mais un exemple qui permet d'approfondir l'analyse.

2. Comme nous l'avons souligné précédemment, une amélioration de la productivité peut résulter d'une hausse des allocations chômage (en l'absence de salaire minimum contraignant). Cependant, nous venons de montrer qu'une hausse du salaire minimum (lorsque celui-ci est contraignant) est susceptible de jouer ce même rôle d'accroissement de la productivité. Ce constat suscite une interrogation à laquelle nous allons essayer de répondre dans la seconde partie de ces simulations: A hausse de la productivité moyenne équivalente, un accroissement des allocations chômage ou, alternativement, une hausse du salaire minimum ont-ils des effets différents sur l'économie, notamment sur le taux de chômage, la distribution des salaires et l'efficacité du marché du travail?

Le surplus collectif permet de comparer l'efficacité de ces mesures sur le fonctionnement du marché du travail. Le critère, $s c$, calculé ici représente le surplus collectif par tête :

$$
s c=(1-u) \bar{y}+u \xi-\theta u c
$$

où $\xi$ représente la production domestique. En notant $b$ les allocations chômage, nous pouvons décomposer ainsi la variable $\zeta: \zeta=\xi+b$

Suivant Marimon et Zilibotti (1999), nous supposons que la fonction d'adéquation prend une forme linéaire:

$$
y(l)=y(0)-\alpha l
$$

Nous retenons les valeurs suivantes des paramètres de la fonction d'appariement: $y(0)=100$ et $\alpha=100$. Ainsi une désadéquation maximale se traduit-elle par une valeur nulle de la productivité du couple employeur/ employé. Il est cependant important de souligner que ce chiffrage est sans influence sur le sens des résultats présentés ci-dessous, seules les valeurs numériques des variables en dépendent.

Suivant Hosios (1990), nous posons comme hypothèse simplificatrice que le taux d'actualisation est égal à zéro $(r=0)$. Cette hypothèse permet de comparer le surplus collectif obtenu dans les différents états stationnaires.

Les autres paramètres prennent les valeurs suivantes : $\beta=0.5$ (ce qui revient à supposer que la négociation de Nash est symétrique), $x=0.75$, $s=0.04, c=150, \xi=10^{5}$.

5 Les résultats des simulations numériques sont robustes et donc préservés pour d'autres valeurs des paramètres. 


\subsection{Les effets d'un accroissement du salaire minimum}

Dans cette première partie des simulations numériques, nous nous bornerons à indiquer le sens de variation des endogènes à la suite d'une augmentation du salaire minimum légal $m$ lorsque celui contraint effectivement la formation des salaires (tableau 2$)^{6}$ :

\begin{tabular}{|l|c|c|c|c|c|c|c|c|c|}
\hline & $\boldsymbol{\theta}$ & $\lambda$ & $\hat{\imath}$ & $\bar{y}$ & $\bar{w}$ & $\boldsymbol{q}$ & $\boldsymbol{p}$ & $\boldsymbol{u}$ & $\boldsymbol{s c}$ \\
\hline$m$ & + & - & - & + & + & - & - & + & + \\
\hline
\end{tabular}

Tableau 2 : Accroissement du salaire minimum

La hausse du salaire minimum entraîne un accroissement du salaire moyen de l'économie. Cependant, cette hausse reste modérée par rapport à celle de la productivité moyenne, il en résulte un accroissement du profit moyen $\bar{y}-\bar{w}$ qui engendre un accroissement des créations d'emplois vacants. La tension sur le marché du travail $\theta$ va alors augmenter.

La hausse de la tension sur le marché du travail n'est néanmoins pas suffisante pour compenser les effets de la plus grande exigence des agents en terme de qualité des appariements (qui se traduit par la baisse de $\lambda$ ) et la probabilité d'obtenir un emploi diminue entrainant une augmentation du taux de chômage. Mais cette baisse de l'emploi est faible, comparativement à l'accroissement de la productivité, et la hausse du salaire minimum augmente la production par tête suffisamment pour que la production totale de l'économie $((1-u) \bar{y})$ s'accroisse.

Le dernier enseignement de cette simulation concerne le surplus collectif $s c$. La hausse du salaire minimum s'avère en effet susceptible d'augmenter ce surplus.

Cependant, la signification de ce résultat doit être clarifiée. Il faut, en effet vérifier que la hausse du surplus collectif provient bien de la plus grande exigence des agents sur la qualité de l'appariement et non pas d'une situation initiale de sur-emploi ${ }^{7}$. Afin d'exclure cette possibilité, nous recalculons le surplus total à $\lambda$ constant; il apparaît que les seules variations de $\theta$ et $u$ entraînent une diminution du surplus collectif. Nous pouvons donc en conclure que c'est bien en améliorant la productivité des associations viables que la hausse du salaire minimum augmente l'efficacité du marché du travail. En d'autres termes, la hausse du surplus social résulte véritablement de la baisse de la désadéquation sur le marché du travail.

6 Les simulations des effets d'une hausse du salaire minimum présentées dans le paragraphe suivant sont une des illustrations possibles de ces variations.

7 Dans un autre article, nous avons montré que la fameuse condition " $\eta=\beta$ " (Hosios (1990)) n'est plus suffisante pour assurer l'efficience de l'équilibre décentralisé (Gavrel, Lages dos Santos et Lebon (2002)) lorsque l'appariement sur le marché du travail dépend à la fois de $\lambda$ et de $\theta$. 


\subsection{Salaire minimum versus allocations chômage}

L'objectif de ce paragraphe est de comparer, à gain de productivité donné, les effets des allocations chômage et du salaire minimum sur le fonctionnement du marché du travail. A cet effet, une situation initiale du marché du travail dans laquelle l'ensemble des salaires se négocient librement et dans laquelle il n'y a pas d'allocations chômage $(b=0)$, nous sert de référence ${ }^{8}$. Nous allons utiliser ce point de départ pour comparer les effets respectifs des deux mesures de politique économique envisagées : (i) l'introduction d'allocations chômage sans salaire minimum contraignant la formation des salaires, (ii) l'introduction d'un salaire minimum touché par une partie des travailleurs en l'absence d'allocations chômage. Dans ce cas, il s'agit de rendre le salaire minimum supérieur au salaire le plus faible résultant de la libre négocation $(w(\lambda))$ dans la situation de référence.

Les valeurs des principales variables dans la situation de référence sont retracées dans le tableau 3 .

\begin{tabular}{|c|c|c|c|c|c|c|c|}
\hline$\theta$ & $\lambda$ & $\bar{y}$ & $\bar{w}$ & $q$ & $p$ & $u$ & $s c$ \\
\hline 0.3728 & 0.3407 & 82.9629 & 74.4443 & 0.7043 & 0.2626 & 0.1322 & 65.9257 \\
\hline
\end{tabular}

Tableau 3 : Situation de référence (sans salaire minimum, ni allocations chômage)

Du fait de la linéarité de la fonction de désadéquation, la distribution des salaires est elle aussi linéaire entre les deux salaires extrêmes: le plus petit salaire qui correspond à la désadéquation maximale, $w(\lambda)=65.9257$, et le plus haut salaire résultant d'une adéquation parfaite entre employeur et employé, $w(0)=82.9629$.

Afin de pouvoir comparer les effets d'une introduction puis d'une hausse des allocations chômage à ceux d'une évolution similaire du salaire minimum, nous calons les hausses simulées des deux variables de politique économique sur un même gain de productivité moyenne. Les hausses successives des allocations chômage présentées sont donc telles qu'elles permettent d'atteindre respectivement les valeurs de 83,84 et 85 pour la productivité moyenne, $\bar{y}$. Les tableaux $4 \mathrm{a}$ et $4 \mathrm{~b}$ présentent les effets des hausses nécessaires des allocations chômage sur l'ensemble des variables de l'économie. 


\begin{tabular}{|l|c|c|c|c|c|c|c|}
\hline & $\bar{y}$ & $\theta$ & $\lambda$ & $\hat{\imath}$ & $\bar{w}$ & $w(\lambda)$ & $w(0)$ \\
\hline$b=0.434$ & 83 & 0.3704 & 0.34 & $\lambda$ & 74.5 & 66 & 83 \\
\hline$b=11.834$ & 84 & 0.3078 & 0.32 & $\lambda$ & 76 & 68 & 84 \\
\hline$b=22.72$ & 85 & 0.2485 & 0.3 & $\lambda$ & 77.5 & 70 & 85 \\
\hline
\end{tabular}

Tableau 4a: Hausses des allocations chômage, avec $m<w(\lambda)$

\begin{tabular}{|l|c|c|c|c|}
\hline & $\boldsymbol{q}$ & $\boldsymbol{p}$ & $\boldsymbol{u}$ & $\boldsymbol{s c}$ \\
\hline$b=0.434$ & 0.7059 & 0.2615 & 0.1327 & 65.9424 \\
\hline$b=11.834$ & 0.75 & 0.2308 & 0.1477 & 66.2522 \\
\hline$b=22.72$ & 0.8 & 0.1988 & 0.1675 & 66.1948 \\
\hline
\end{tabular}

Tableau 4b : Hausses des allocations chômage, avec $m<w(\lambda)($ suite)

Les tableaux 5a et 5b retracent les effets d'une introduction puis d'une hausse du salaire minimum contraignant la formation des salaires et correspondant aux mêmes gains de productivité moyenne.

\begin{tabular}{|c|c|c|c|c|c|c|c|}
\hline & $\bar{y}$ & $\theta$ & $\lambda$ & $\hat{\imath}$ & $\bar{w}$ & $w(\lambda)$ & $w(0)$ \\
\hline$m=66$ & 83 & 0.3729 & 0.34 & 0.3394 & 74.4707 & $m$ & 83 \\
\hline$m=68$ & 84 & 0.3737 & 0.32 & 0.3033 & 75.1863 & $m$ & 84 \\
\hline$m=70$ & 85 & 0.3751 & 0.3 & 0.2665 & 75.9189 & $m$ & 85 \\
\hline
\end{tabular}

Tableau 5a : Hausses du salaire minimum à $b=0$

\begin{tabular}{|l|c|c|c|c|}
\hline & $\boldsymbol{q}$ & $\boldsymbol{p}$ & $\boldsymbol{u}$ & $\boldsymbol{s c}$ \\
\hline$m=66$ & 0.7035 & 0.2623 & 0.1323 & 65.9434 \\
\hline$m=68$ & 0.6808 & 0.2544 & 0.1359 & 66.3290 \\
\hline$m=70$ & 0.6607 & 0.2445 & 0.1406 & 66.6510 \\
\hline
\end{tabular}

Tableau 5b : Hausses du salaire minimum à $b=0$ (suite)

Bien que les deux instruments permettent d'obtenir les mêmes résultats en terme de réduction de la désadéquation entre employeur et employé et donc d'amélioration de la productivité, les effets sur le reste des variables de l'économie diffèrent. Cette différence provient de ce que la hausse des allocations chômage qui affecte directement l'ensemble des salaires négociés, 
engendre une forte augmentation du salaire moyen et donc une baisse de la profitabilité moyenne des emplois. Il en résulte une baisse des créations d'entreprises et de la tension sur le marché du travail qui provoque à son tour un fort accroissement du taux de chômage. A l'inverse, le salaire minimum n'a pas d'effet direct sur les salaires négociés et leur augmentation, limitée, ne provient que de la hausse de la tension sur le marché du travail qui résulte elle-même d'une hausse de la profitabilité moyenne des emplois.

A cause de la différence d'accroissement du taux de chômage, l'augmentation du surplus collectif permise par l'introduction des allocations chômage est beaucoup plus faible que celle obtenue avec le salaire minimum De plus, ainsi que le montrent les résultats numériques (tableau 4b), la tendance en vient même à s'inverser dès que les allocations chômage deviennent un peu plus élevées, car la hausse de la productivité n'est plus suffisante pour compenser l'augmentation du taux de chômage. En revanche, le salaire minimum permet un accroissement du surplus collectif jusqu'à des niveaux nettement plus importants.

Comme l'illustrent les graphiques 3 et 4 , l'introduction puis la hausse d'un salaire minimum écrasent la distribution des salaires et réduisent donc les inégalités salariales beaucoup plus que ne font les allocations chômage. Il faut cependant souligner que les allocations chômage permettent de réduire l'inégalité de revenu entre salariés et chômeurs, alors que la hausse du salaire minimum tend au contraire à accroître cette inégalité. Cette observation doit conduire à relativiser la préférence pour le salaire minimum comme instrument de politique économique qui résulterait de la seule prise en compte des effets sur le surplus. S'il privilégie l'équité, le gouvernement peut y voir une raison suffisante pour lui adjoindre des allocations chômage.

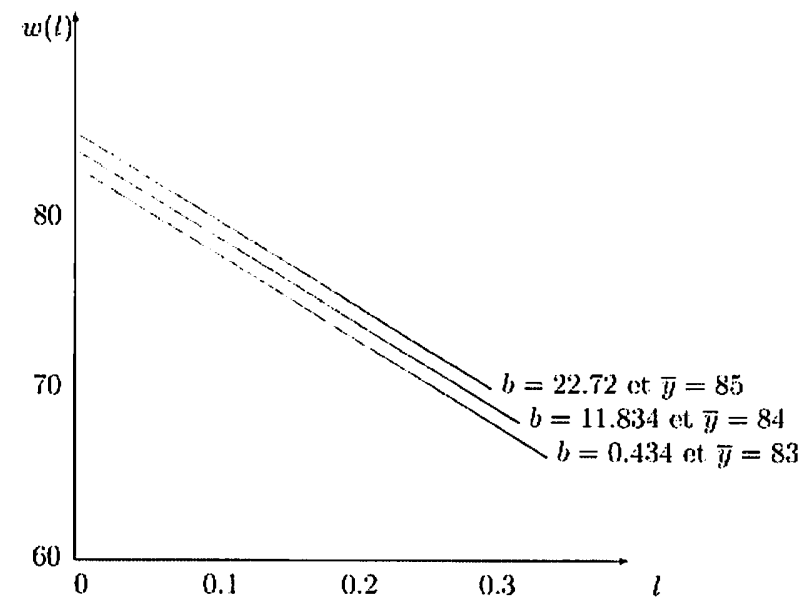

Figure 3 : Allocations chômage et évolution de la distribution des salaires en fonction de la productivité moyenne 


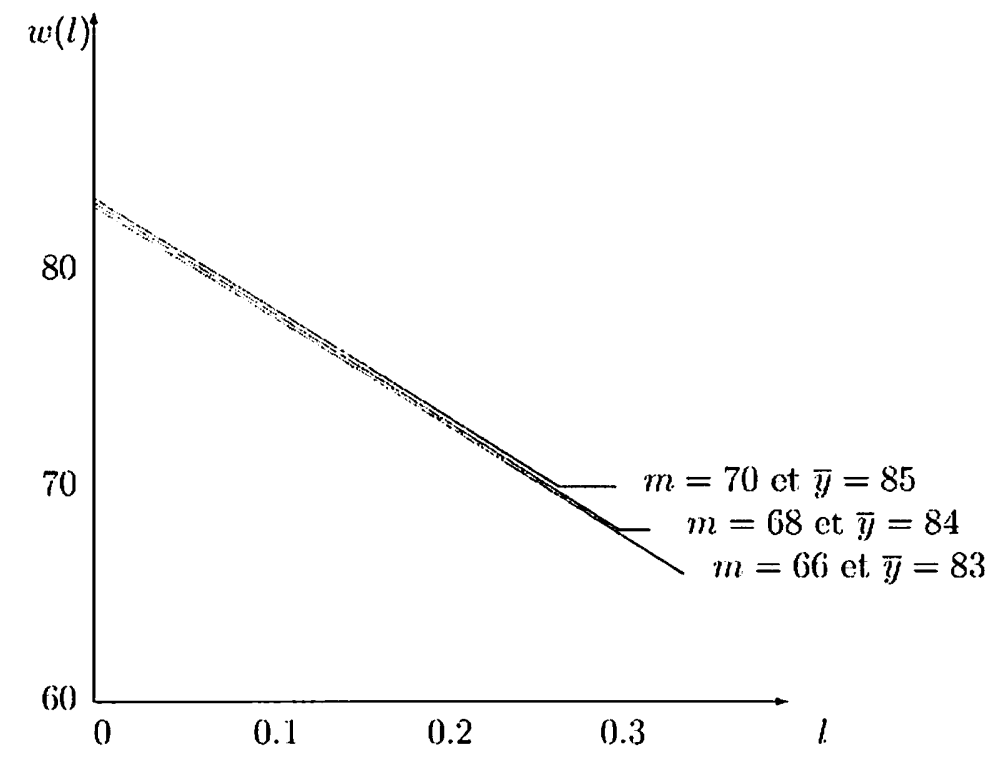

Figure 4 : Salaire minimum et évolution de la distribution des salaires en fonction de la productivité moyenne

\section{$5 \quad$ Remarques finales}

Deux principaux enseignements émergent de ce réexamen théorique des effets de la législation du travail. D'une part, si l'on s'intéresse aux effets des allocations chômage, on ne peut le faire en ignorant les contraintes légales qui pèsent éventuellement sur la formation des salaires. D'autre part, la régulation des salaires donne au gouvernement un instrument simple et puissant pour contrôler la désadéquation et la productivité du travail.

L'introduction du salaire minimum ou, en son absence, la hausse des allocations chômage peuvent donc améliorer le fonctionnement du marché du travail en empêchant que soient constituées les plus mauvaises associations entre entreprises et salariés. Dans notre modèle, ces associations peu productives sont d'autant plus nombreuses que les entreprises embauchent le premier candidat acceptable et pas forcément le meilleur, et que les travailleurs qui ont un emploi et les entreprises dont l'emploi est occupé ne peuvent pas poursuivre leur recherche d'un meilleur partenaire. Ces deux aspects constituent les limites de notre modèle. Une possibilité de recherche en emploi ("on-the-job-search") et un processus d'embauche plus efficace qui diminueraient la part des emplois à faible productivité, viendraient probablement réduire l'efficacité des instruments de la politique économique. Ces questions devront faire l'objet de recherches futures. 


\section{Bibliographie}

Acemoglu D. et R. Shimer (2000). "Productivity gains from unemployment insurance", European Economic Review, vol. 44, pp. 1195-1224.

Aghion P. et P. Howitt (1992). "A model of growth through creative destruction", Econometrica, vol. 60, pp. 323-351.

Albrecht J., P. Gautier et S. Vroman (2003). "Matching with multiple applications", Economics Letters, vol. 78, n¹, pp. 67-70.

Amine S., F. Gavrel et I. Lebon (2007). "Jobs specialization vs unemployment: More on the productivity effect of unemployment benefits", Spanish Economic Review, vol. 9, pp. 177-191.

Blanchard O. et P. Diamond (1989). "The Beveridge curve", Brookings Papers on Economic Activity, vol. 1, pp. 1-76.

Blanchard O. et S. Fisher (1989). Lectures on Macroeconomics, MIT Press.

Card D. et A. Krueger (1994). "Minimum wages and employment: a case study of the fast-food industry in New Jersey and Pennsylvania", American Economic Review, vol. 84, pp. 772-793.

Card D. et A. Krueger (1995). Myth and measurement, the new economics of the minimum wage, Princeton University Press.

Gavrel F. (2001). "Differentiation of skills, firms' recruitment strategy and unemployment benefits in an efficiency wage model", De Economist, vol. 149, pp. 347-364.

Gavrel F., P. Lages dos Santos et I. Lebon (2002). $\beta=\eta$ is not enough: Reexamining the efficiency of the labour market in a matching model with differentiation of skills, Mimeo.

Gavrel F. et I. Lebon (2004). Providing Pissarides' stochastic job matching model with microeconomic foundations. A reconsideration of Marimon and Zilibotti (1999) circle model, Mimeo.

Hall R. (1977). "An aspect of the economic role of unemployment", in: Harcourt G. (ed), The Microeconomic Foundations of Macroeconomics, Maclillan.

Hosios D. (1990). "On the efficiency of matching and related models of search and unemployment", Review of Economic Studies, vol. 55, pp. 279-298.

Marimon R. at F.Zilibotti (1999). "Unemployment VS. mismatch of talents: Reconsidering unemployment benefits", Economic Journal, vol. 109, pp. 266-291.

Mc Kenna C. (1985). Uncertainty and the labour market, Harvester Press.

Petrongolo B. et C. Pissarides (2001). "Looking into the black box : A survey of the matching function", Journal of Economic Literature, vol. 39 , pp. $390-431$. 
Pissarides C. (1979). "Job matchings with state employment agencies and random search", Economic Journal, vol. 89, pp. 818-833.

Pissarides C. (1984). "Efficient job rejection", Economic Journal, vol. 94, pp. $97-108$.

Pissarides C. (2000). Equilibrium Unemployment Theory, $2^{\mathbf{c}}$ ed, MIT Press.

Rebitzer J. et L. Taylor (1995). "The consequences of minimum wage laws: some new theoretical ideas", Journal of Public Economics, vol. 56, pp. 245-255.

Salop S. (1979). "Monopolistic competition with outside goods", Bell Journal of Economics, vol. 10, pp. 141-156.

Stigler G. (1946). "The economics of minimum wage legislation", American Economic Review, vol. 36, pp. 358-365.

\section{Annexe A}

Le modèle des "urnes et boules" ("urn-ball model") avec différenciation des qualifications

L'objet de cette annexe est de déterminer la probabilité de pourvoir un emploi vacant sur le marché du travail. Un emploi vacant est pourvu si, et seulement si, au moins l'un des candidats à cet emploi est situé à une distance inférieure à la limite $\lambda$ de l'entreprise.

Pour $\lambda \leq x$, la probabilité qu'aucun chômeur employable (ces chômeurs sont au nombre de $\lambda U$ ) n'ait candidaté à ce poste, est donné par :

$$
\left(\frac{x V-1}{x V}\right)^{\lambda U}=\exp \left[\lambda U \ln \left(1-\frac{1}{x V}\right)\right]
$$

D'où $\left(1-\exp \left[\lambda U \ln \left(1-\frac{1}{x V}\right)\right]\right)$ représente la probabilité d'avoir au moins un candidat employable.

Parce que les chômeurs et les emplois vacants sont "très nombreux", on obtient :

$$
q=1-e^{-\frac{\lambda U}{x V}}=1-e^{-\frac{\lambda}{x \cdot \theta}}
$$

\section{Annexe B}

Le comportement de la fonction $p(\theta, \lambda)$

Nous avons :

$$
p(\theta, \lambda)=\theta q(\theta, \lambda)=\theta\left(1-e^{-\frac{\lambda}{x \theta}}\right)
$$


La dérivée de $p($.$) par rapport à \theta$ est donnée par :

$$
1-e^{-\frac{\lambda}{x \theta}}-\frac{\lambda}{x \theta} e^{-\frac{\lambda}{x \theta}}
$$

Il est facile de montrer que cette dérivée prend des valeurs comprises dans un intervalle |0,1|. Donc, la probabilité $p$ est une fonction croissante de la tension sur le marché du travail $\theta$.

\section{Annexe C}

\section{Détermination de l'équilibre sans salaire minimum} contraignant la formation des salaires

Dans le cas où tous les salaires sont librement négociés, $\lambda$ est égal à $\hat{l}(\lambda=\hat{l})$. Comme dans la version du modèle avec salaire minimum, la valeur de $\lambda$ correspond à la distance qui annule le surplus du couple employeur/ employé :

$$
W_{E}(\lambda)-W_{U}+J_{F}(\lambda)-J_{V}=0
$$

La négociation salariale (équation (15)) qui est la règle pour tous les niveaux de désadéquation impose alors :

$$
W_{E}(\lambda)-W_{U}=0 \Leftrightarrow J_{F}(\lambda)=0 \Leftrightarrow w(\lambda)=y(\lambda)
$$

$C$ 'est à partir de cette relation qui vient se substituer à l'équation (17), que se détermine la valeur de $\lambda$ en fonction de $\theta$. Etant données les relations (5) et (6), on a :

$$
W_{E}(\lambda)-W_{U}=0 \Leftrightarrow r W_{U}=R y(\lambda)
$$

Avec les équations (6) et (14), on obtient :

$$
r W_{U}=R b+p\left(\bar{W}_{E}-W_{U}\right)=R \zeta+p \frac{\beta}{(1-\beta)} \bar{J}_{F}=R \zeta+R \frac{p}{q(1-\beta)} c
$$

Il apparaît donc finalement :

$$
y(\lambda)=\zeta+\theta \frac{\beta}{(1-\beta)} c
$$

En combinant cette relation avec l'équation (30) ( $\lambda$ étant égal à $\hat{l}$ ), on obtient le couple $\left(\lambda^{*}, \theta^{*}\right)$ qui définit l'équilibre. Les autres variables de l'économie s'en déduisent. C'est à partir de ces relations qu'est construit le programme de simulations "sans salaire minimum contraignant la formation des salaires" 9 .

9 Les programmes de simulations (réalisées sous MATLAB) sont à la disposition des lecteurs intéressés 